\title{
Correction to: The Hybrid High-Order Method for Polytopal Meshes
}

\section{Correction to:}

\section{A. Di Pietro, J. Droniou,}

The Hybrid High-Order Method for Polytopal Meshes, MS\&A 19, https://doi.org/10.1007/978-3-030-37203-3

Inadvertently, the author name was captured in the metadata of the chapters as Daniele Di Antonio Pietro, whereas it should be Daniele Antonio Di Pietro. This has been corrected now in the metadata. 